\title{
MicroRNA-432 is downregulated in cervical cancer and directly targets FN1 to inhibit cell proliferation and invasion
}

\author{
SHANZONG WANG ${ }^{1}$, BAOHONG GAO $^{2}$, HAILIN YANG $^{3}$, XUEJIAN LIU $^{3}$, XIA WU $^{3}$ and WEIJUAN WANG ${ }^{2}$ \\ Departments of ${ }^{1}$ Pathology, ${ }^{2}$ Gynecology and ${ }^{3}$ Oncology, \\ The Third People's Hospital of Linyi, Linyi, Shandong 276023, P.R. China
}

Received October 15, 2018; Accepted April 4, 2019

DOI: $10.3892 / \mathrm{ol} .2019 .10403$

\begin{abstract}
Numerous studies have identified the dysregulation of microRNAs (miRNAs) in cervical cancer, and dysregulated miRNAs are involved in regulating a number of tumourassociated biological behaviours. Therefore, investigating the roles of cervical cancer-associated miRNAs and the underlying molecular mechanisms is essential for the development of novel diagnostic biomarkers and effective therapeutic targets. MicroRNA-432 (miR-432) dysregulation has been revealed to be implicated in the carcinogenesis and progression of a number of types of human cancer. However, the effects and underlying molecular mechanisms of miR-432 in cervical cancer have yet to be elucidated. In the present study, miR-432 expression was determined using reverse transcription-quantitative polymerase chain reaction. The results revealed that miR-432 was expressed at low levels in cervical cancer tissues and cell lines. Decreased miR-432 expression was significantly associated with the International Federation of Gynecology and Obstetrics stage, myometrium invasion and lymph node metastasis of patients with cervical cancer. Following transfection with miR-432 mimic, the expression of miR-432 was significantly upregulated in cervical cancer cells. Upregulation of miR-432 expression restricted the proliferation and invasion of cervical cancer cells. Bioinformatics analysis followed by luciferase reporter assays revealed that fibronectin 1 (FN1) was a direct target gene of miR-432 in cervical cancer cells. In addition, FN1 was upregulated in cervical cancer tissues and was inversely correlated with miR-432 levels. Furthermore, miR-432 upregulation decreased the expression levels of FN1 in cervical cancer cells at the mRNA and protein levels. Furthermore, silencing of FN1 could stimulate the tumour suppressor effects of miR-432 upregulation in cervical cancer cells. In addition, restored FN1 expression neutralized the
\end{abstract}

Correspondence to: Professor Weijuan Wang, Department of Gynecology, The Third People's Hospital of Linyi, 117 Huaxia Road, Linyi, Shandong 276023, P.R. China

E-mail:wj_wanglinyi@126.com

Key words: microRNA-432, cervical cancer, fibronectin 1, proliferation, invasion effects of miR-432 overexpression in cervical cancer cells. The results of the present study indicate that miR-432 is a tumour suppressor that can restrain the aggressive phenotype of cervical cancer cells by directly targeting FN1, suggesting that this miRNA may be developed as an effective therapeutic strategy for patients with cervical cancer.

\section{Introduction}

Cervical cancer, one of the most common types of gynaecological malignancy, is the fourth most frequent cause of cancer-associated mortality among females worldwide (1). The incidence of cervical cancer is decreasing each year in developed countries; unfortunately, however, it continues to increase each year in developing countries (2). It is estimated that there are $\sim 500,000$ novel cases and $>250,000$ mortalities caused by cervical cancer per year globally (3). In recent years, impressive progress has been made in diagnostic techniques and therapeutic approaches; however, cervical cancer remains a public health concern worldwide, and the clinical outcomes of patients with cervical cancer remain unsatisfactory (4). Multiple factors, such as local recurrence, distant metastasis and diagnostic delays, are mainly responsible for the poor prognosis of patients with cervical cancer (5); however, the specific underlying molecular mechanism remains largely unknown. Thus, further studies are required to elucidate the molecular mechanisms underlying cervical carcinogenesis and progression to develop promising therapeutic methods for treating patients with cervical cancer.

MicroRNAs (miRNAs) are a subset of endogenous, non-coding and small RNA molecules (2). These 17-23-nucleotide-long RNAs are able to post-transcriptionally modulate the expression of relevant genes via base-pairing with the 3'-untranslated regions (3'-UTRs) of their target genes, thereby resulting in either translation suppression and/or mRNA cleavage (6). To date, $>1,500$ mature miRNAs have been identified in the human genome, and these miRNAs can regulate $\sim 30 \%$ of human protein-coding genes (6). miRNA aberrations have been identified in various types of malignancy, including cervical cancer (7). It has been demonstrated that a number of miRNAs are abnormally expressed during the occurrence and progression of cervical cancer (8). For example, miR-143 (9), miR-152 (10) and miR-506 (11) were downregulated in cervical cancer, whereas miR-21 (12), miR-224 (13) and miR-1297 (14) 
were expressed at high levels. Recent evidence has indicated that deregulated miRNAs may serve crucial roles in cervical cancer initiation and progression by regulating a wide range of biological functions (15-17). Therefore, miRNAs may be effective therapeutic targets for the treatment of patients with cervical cancer.

miR-432 dysregulation has been demonstrated to be implicated in the carcinogenesis and progression of numerous types of human cancer, including lung adenocarcinoma (18), neuroblastoma (19), hepatocellular carcinoma (20) and prostate cancer (21). However, the effects and underlying molecular mechanisms of miR-432 in cervical cancer have yet to be elucidated. The objectives of the present study were to detect the expression of miR-432 in cervical cancer and determine its clinical significance. The effects of miR-432 in cervical cancer cells and the potential underlying molecular mechanism of these effects were also investigated.

\section{Materials and methods}

Clinical tissue specimens. A total of 47 pairs of cervical cancer tissues and matched adjacent normal tissues were collected from female patients (age range, 49-65 years) who received surgery at The Third People's Hospital of Linyi (Linyi, China) between April 2015 and August 2017. All tissues were obtained using microdissection. None of these patients had been treated with any pre-operative chemotherapy or radiotherapy. Once obtained, tissues were immediately frozen in liquid nitrogen and then stored at $-80^{\circ} \mathrm{C}$ for further study. Patients that had been treated with pre-operative chemotherapy or radiotherapy were excluded from the study. All patients with cervical cancer were divided into either a low miR-432 expression group or a high miR-432 expression group using the median value of miR-432 expression (0.45) as the cut-off. The International Federation of Gynecology and Obstetrics (FIGO) stage is currently the most commonly used staging system for cancer of the uterine cervix worldwide (22). Tumours are classified by roman numerals from I to $\mathrm{IV}$, representing a range from precancerous or in situ to highly malignant. Classification subdivisions are indicated by letters and numbers (22). The present study was approved by the Ethics Committee of The Third People's Hospital of Linyi. All patients provided written informed consent and were apprised of the aims of the study.

Cell lines. Three human cervical cancer cell lines, HeLa, CaSki and SiHa, were purchased from the Cell Bank of the Chinese Academy of Sciences (Shanghai, China). The normal human cervix epithelial cell line, Ect1/E6E7, was purchased from the American Type Culture Collection (ATCC; Manassas, VA, USA). All cell lines were cultured in Dulbecco's modified Eagle's medium (DMEM) containing 10\% foetal bovine serum (FBS), $100 \mathrm{U} / \mathrm{ml}$ penicillin and $100 \mu \mathrm{g} / \mathrm{ml}$ streptomycin (all purchased from Invitrogen; Thermo Fisher Scientific, Inc., Waltham, MA, USA) and grown in a humidified incubator supplied with $5 \% \mathrm{CO}_{2}$ at $37^{\circ} \mathrm{C}$.

Oligonucleotide transfection. Synthetic miR-432 mimic and negative control miRNA mimic (miR-NC) were obtained from Guangzhou RiboBio Co., Ltd. (Guangzhou, China). For silencing of fibronectin 1 (FN1) expression, a small interfering
RNA (siRNA) against FN1 (FN1 siRNA) and negative control siRNA (NC siRNA) were chemically synthesized by GeneCopoeia, Inc. (Rockville, MD, USA). The FN1 expression plasmid pcDNA3.1-FN1 (pc-FN1) and blank pcDNA3.1 plasmid were generated by Shanghai GenePharma Co., Ltd. (Shanghai, China). HeLa and SiHa cells in the exponential growth phase were collected and seeded into 6-well plates at a density of $6 \times 10^{6}$ cells/well 1 day before transfection. Cells were transfected with miRNA mimic (100 pmol), siRNA $\left(100\right.$ pmol) or plasmid $(4 \mu \mathrm{g})$ using Lipofectamine ${ }^{\circledR} 2000$ reagent (Invitrogen; Thermo Fisher Scientific, Inc.) according to the manufacturer's protocol. The transfected HeLa and $\mathrm{SiHa}$ cells were harvested and used for the detection of miR-432 and FN1 protein expression at 48 and $72 \mathrm{~h}$ after transfection, respectively.

RNA extraction and reverse transcription-quantitative polymerase chain reaction $(R T-q P C R)$. Total RNA was isolated from cell lines and tissues using TRIzol ${ }^{\circledR}$ reagent (Invitrogen; Thermo Fisher Scientific, Inc.), and the concentration of isolated total RNA was determined using a NanoDrop ${ }^{\text {TM }} 1000$ spectrophotometer (Thermo Fisher Scientific, Inc.). To quantify miR-432 expression, total RNA was converted into cDNA using the TaqMan MicroRNA RT kit (Applied Biosystems; Thermo Fisher Scientific, Inc.). The temperature protocol for reverse transcription was as follows: $16^{\circ} \mathrm{C}$ for $30 \mathrm{~min}, 42^{\circ} \mathrm{C}$ for $30 \mathrm{~min}$ and $85^{\circ} \mathrm{C}$ for $5 \mathrm{~min}$. Next, qPCR was performed using the TaqMan MicroRNA PCR kit (Applied Biosystems; Thermo Fisher Scientific, Inc.). The temperature protocol for qPCR was as follows: $50^{\circ} \mathrm{C}$ for $2 \mathrm{~min}, 95^{\circ} \mathrm{C}$ for $10 \mathrm{~min}$; 40 cycles of denaturation at $95^{\circ} \mathrm{C}$ for $15 \mathrm{sec}$; and annealing/extension at $60^{\circ} \mathrm{C}$ for $1 \mathrm{~min}$. For FN1 mRNA expression analysis, cDNA was generated from total RNA using the PrimeScript RT Reagent kit followed by qPCR using the SYBR ${ }^{\circledR}$ Premix Ex $\mathrm{Taq}^{\mathrm{TM}}$ kit (both from Takara Biotechnology Co., Ltd., Dalian, China). The temperature protocol for reverse transcription was as follows: $37^{\circ} \mathrm{C}$ for $15 \mathrm{~min}$ and $85^{\circ} \mathrm{C}$ for $5 \mathrm{sec}$. The qPCR was performed with cycling conditions as follows: $5 \mathrm{~min}$ at $95^{\circ} \mathrm{C}$, followed by 40 cycles of $95^{\circ} \mathrm{C}$ for $30 \mathrm{sec}$ and $65^{\circ} \mathrm{C}$ for $45 \mathrm{sec}$. U6 small nuclear RNA and GAPDH were used as internal references for miR-432 and FN1 mRNA expression, respectively. Relative gene expression was quantified using the $2^{-\Delta \Delta \mathrm{Cq}}$ method (23). The primers were designed as follows: miR-432 forward, 5'-AACGAGACGACGACA GACT-3' and reverse, 5'-CTTGGAGTAGGTCATTGGGT-3'; U6 forward, 5'-GCTTCGGCAGCACATATACTAAAAT-3' and reverse, 5'-CGCTTCACGAATTTGCGTGTCAT-3'; FN1 forward, 5'-CAGTGGGAGACCTCGAGAAG-3' and reverse, 5'-TCCCTCGGAACATCAGAAAC-3'; and GAPDH forward, 5'-GCTGGCGCTGAGTACGTCGTGGAGT-3' and reverse, 5'-CACAGTCTTCTGGGTGGCAGTGATGG-3'.

Cell Counting Kit-8 (CCK-8) assay. The influence of miR-432 on cervical cancer cell proliferation was evaluated using the CCK-8 assay. Briefly, transfected HeLa and SiHa cells were collected after $24 \mathrm{~h}$ of incubation, and $3 \times 10^{3}$ cells/well were inoculated into 96-well plates in triplicate. Following incubation for $0,24,48$ or $72 \mathrm{~h}$ at $37^{\circ} \mathrm{C}$ with $5 \% \mathrm{CO}_{2}$, a CCK-8 assay was performed by adding $10 \mu \mathrm{l}$ CCK- 8 solution (Dojindo Molecular Technologies, Inc., Kumamoto, Japan) to each well. 
Cells were then incubated at $37^{\circ} \mathrm{C}$ for an additional $2 \mathrm{~h}$. Finally, the optical density at a wavelength of $450 \mathrm{~nm}$ was determined using a spectrophotometer (Victor X; PerkinElmer, Inc., Waltham, MA, USA).

Transwell Matrigel invasion assay. A Transwell Matrigel invasion assay was used to identify the invasive capacity of cervical cancer cells. To this end, transfected cells were harvested after $48 \mathrm{~h}$ of incubation and suspended in FBS-free DMEM. In total, $5 \times 10^{4}$ cells were placed in the upper compartment of Transwell inserts that were coated with Matrigel (both purchased from BD Biosciences, San Jose, CA, USA). DMEM supplemented with $20 \%$ FBS was added to the lower compartments as a chemoattractant. After $24 \mathrm{~h}$ of incubation, non-invasive cells were gently wiped off using a cotton swab. The invasive cells on the bottom side of the membrane were fixed with $4 \%$ paraformaldehyde at $37^{\circ} \mathrm{C}$ for $15 \mathrm{~min}$ and stained with $0.1 \%$ crystal violet at $37^{\circ} \mathrm{C}$ for $30 \mathrm{~min}$. The invasive ability of cervical cancer cells was assessed by counting the number of invasive cells in five randomly selected visual fields from each insert using a light microscope (x200 magnification; Olympus Corporation, Tokyo, Japan).

Bioinformatics analysis. The databases TargetScan (targetscan. org) and miRDB (mirdb.org) software were used to predicate the putative targets of miR-432.

Luciferase reporter assay. The wild-type (WT) and mutant (MUT) 3'-UTRs of FN1 were amplified by and purchased from Shanghai GenePharma Co., Ltd., and cloned into pMIR (Ambion; Thermo Fisher Scientific, Inc.), generating the pMIR-WT-FN1-3'-UTR and pMIR-MUT-FN1-3'-UTR plasmids, respectively. For the reporter assay, cells in the exponential growth phase were plated on 24-well plates with a density of $1.5 \times 10^{5}$ cells/well. miR-432 mimic (50 pmol) or miR-NC (50 pmol) in combination with pMIR-WT-FN1-3'-UTR $(0.2 \mu \mathrm{g})$ or pMIR-MUT-FN1-3'-UTR $(0.2 \mu \mathrm{g})$ were co-transfected into cells using Lipofectamine 2000 reagent, according to the manufacturer's protocol.

Luciferase activity was determined using a dual-luciferase reporter assay system (Promega Corporation, Madison, WI, USA) $48 \mathrm{~h}$ after transfection. The activity of firefly luciferase was normalized to that of Renilla luciferase.

Western blot analysis. Tissue samples and cells were washed twice with PBS and lysed with radioimmunoprecipitation assay lysis buffer (Shanghai Qcbio Science \& Technologies Co., Ltd., Shanghai, China). The concentration of total protein was determined using a bicinchoninic acid kit (Beyotime Institute of Biotechnology, Haimen, China). Equal amounts of total protein $(30 \mu \mathrm{g})$ were separated by SDS-PAGE (10\% gel) and then transferred onto polyvinylidene difluoride membranes (Beyotime Institute of Biotechnology). Following blocking with 5\% skimmed milk suspended in Tris-buffered saline-containing Tween-20 (TBST) at room temperature for $2 \mathrm{~h}$, the membranes were incubated overnight at $4^{\circ} \mathrm{C}$ with primary antibodies as follows: Rabbit anti-human FN1 antibody (catalogue no. 15613-1-AP; 1:200 dilution; ProteinTech Group, Inc., Chicago, IL, USA) and rabbit anti-human GAPDH antibody (catalogue no. ab181603; 1:1,000 dilution; Abcam,
Cambridge, UK). Following extensive washes with TBST, horseradish peroxidase-conjugated goat anti-rabbit secondary antibody (catalogue no. ab6721; 1:5,000 dilution; Abcam, Cambridge, UK) was added for $2 \mathrm{~h}$ at room temperature. Finally, the immunoreactive proteins were visualized using an enhanced chemiluminescence protein detection kit (Pierce; Thermo Fisher Scientific, Inc.). GAPDH was used as a loading control.

Statistical analysis. Data are presented as the mean \pm standard deviation. Student's t-test was used to compare the difference between two groups. The differences between multiple groups were evaluated using one-way analysis of variance, followed by Tukey's post hoc test. A $\chi^{2}$ test was applied to investigate the association between miR-432 and the clinical characteristics in patients with cervical cancer. The correlation between expression levels of miR-432 and FN1 mRNA in cervical cancer tissues was assessed using Spearman's correlation analysis. $\mathrm{P}<0.05$ was considered to indicate a statistically significant difference.

\section{Results}

miR-432 expression is decreased and significantly associated with certain clinicopathological features of cervical cancer. To investigate the clinical value of miR-432 in cervical cancer, the expression levels of miR-432 in 47 pairs of cervical cancer tissues and matched adjacent normal tissues were determined using RT-qPCR. The results revealed a significant decrease in the expression of miR-432 in cervical cancer tissues compared with in adjacent normal tissues $(\mathrm{P}<0.05$; Fig. $1 \mathrm{~A})$. The clinical significance of miR-432 expression in cervical cancer was then investigated. All patients with cervical cancer were divided into either a low miR-432 expression group or a high miR-432 expression group using the median value of miR-expression $(0.45)$ as the cut-off. Decreased miR-432 expression was revealed to be significantly associated with the FIGO stage $(\mathrm{P}=0.020)$, myometrium invasion $(\mathrm{P}=0.001)$ and lymph node metastasis $(\mathrm{P}=0.008)$. However, no significant association was identified with age or tumour size (both $\mathrm{P}>0.05$; Table I). In addition, the expression level of miR-432 in three human cervical cancer cell lines (HeLa, CaSki and $\mathrm{SiHa}$ ) and a normal human cervix epithelial cell line (Ect1/E6E7) was determined. RT-qPCR analysis revealed that miR-432 was expressed at low levels in all three cervical cancer cell lines relative to Ect1/E6E7 ( $\mathrm{P}<0.05$; Fig. 1B). These results suggest that downregulation of miR-432 may be involved in the development and progression of cervical cancer.

Ectopic miR-432 expression inhibits the proliferation and invasion of cervical cancer cells. HeLa and SiHa cell lines exhibited the greatest decrease in miR-432 expression among the three cervical cancer cell lines, therefore these two cervical cancer cell lines were selected for subsequent functional assays. To identify the specific roles of miR-432 in cervical cancer cells, miR-432 mimic or miR-NC were introduced into HeLa and SiHa cells. RT-qPCR analysis verified that miR-432 was markedly overexpressed in miR-432 mimic-transfected HeLa and SiHa cells compared with cells transfected with miR-NC and non-transfected cells 

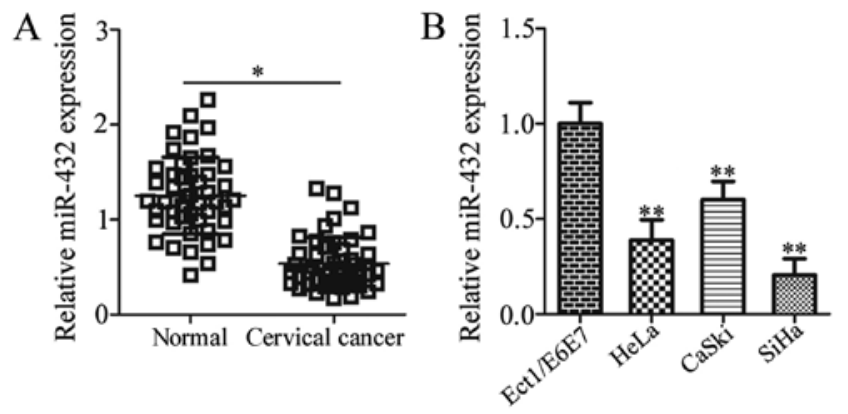

Figure 1. miR-432 is downregulated in cervical cancer. (A) Total RNA was isolated from cervical cancer tissues and matched adjacent normal tissues. The expression level of miR-432 was determined using RT-qPCR. ${ }^{*} \mathrm{P}<0.05$ vs. adjacent normal tissues. (B) RT-qPCR was performed to evaluate miR-432 expression in three human cervical cancer cell lines (HeLa, CaSki and $\mathrm{SiHa}$ ) and a normal human cervix epithelial cell line (Ect1/E6E7). ${ }^{* *} \mathrm{P}<0.05$ vs. Ect1/E6E7. miR-432, microRNA-432; RT-qPCR, reverse transcription-quantitative polymerase chain reaction.

$(\mathrm{P}<0.05$; Fig. 2A). In addition, no significant difference in miR-432 expression was observed between the miR-NC and non-transfected groups $(\mathrm{P}<0.05)$. A CCK-8 assay was performed in order to investigate the effect of miR-432 overexpression on the proliferation of cervical cancer cells. The results indicated that when HeLa and SiHa cells were transfected with miR-432 mimic, the proliferative ability was significantly decreased compared with that of miR-NC groups at 48 and $72 \mathrm{~h}(\mathrm{P}<0.05$; Fig. 2B). Furthermore, a Transwell Matrigel invasion assay was performed to investigate the effect of miR-432 on cervical cancer cell invasion. It was revealed that enforced miR-432 expression significantly inhibited the invasion of HeLa and SiHa cells $(\mathrm{P}<0.05$; Fig. 2C). These results suggest that miR-432 may have anti-proliferative and anti-invasive roles in cervical cancer cells.

FN1 is a direct target gene of miR-432 in cervical cancer cells. To further illustrate the molecular mechanisms by which miR-432 inhibits cervical cancer cell proliferation and invasion, a bioinformatics analysis was performed in order to predict the potential targets of miR-432. A highly conserved putative binding site was revealed at 154-160 bp of the FN1-3'-UTR (Fig. 3A). FN1 was selected for further investigation as this gene was considered to have a role in the formation and progression of cervical cancer (24-27). To confirm this prediction, luciferase reporter plasmids were chemically synthesized and co-transfected with miR-432 mimic or miR-NC into HeLa and SiHa cells. The results of the luciferase reporter assays in the present study indicated that miR-432 overexpression notably decreased the luciferase activity of the plasmid harbouring WT-FN1-3'-UTR $(\mathrm{P}<0.05)$; however, the suppression of luciferase activity was eliminated when HeLa and SiHa cells were co-transfected with miR-432 mimic and luciferase plasmid containing MUT-FN1-3'-UTR (Fig. 3B).

RT-qPCR was performed to determine FN1 expression in 47 pairs of cervical cancer tissues and matched adjacent normal tissues. The data revealed that the expression level of FN1 mRNA was significantly higher in cervical cancer tissues compared with in adjacent normal tissues $(\mathrm{P}<0.05$;
Table I. Association between miR-432 and the clinicopathological features of patients with cervical cancer.

\begin{tabular}{lrrr}
\hline & \multicolumn{2}{c}{ miR-432 level } & \\
\cline { 2 - 3 } Clinicopathological feature & Low & High & P-value \\
\hline Age, years & & & 0.534 \\
$<45$ & 9 & 6 & \\
$\geq 45$ & 15 & 17 & \\
Tumor size, cm & & & 0.380 \\
$<4$ & 16 & 12 & \\
$\geq 4$ & 8 & 11 & \\
FIGO stage & & & 0.020 \\
I-II & 7 & 15 & \\
III-IV & 17 & 8 & \\
Myometrium invasion & & & 0.001 \\
$\quad<1 / 2$ & 7 & 18 & \\
$\geq 1 / 2$ & 17 & 5 & \\
Lymph node metastasis & & & 0.008 \\
$\quad$ Negative & 8 & 17 & \\
Positive & 16 & 6 & \\
\hline
\end{tabular}

miR-432, microRNA-432; FIGO, Federation of Gynecology and Obstetrics.

Fig. 3C). Notably, FN1 mRNA expression levels in cervical cancer tissues were inversely correlated with miR-432 expression levels ( $\mathrm{r}=-0.5383, \mathrm{P}<0.0001$; Fig. 3D). Furthermore, re-expression of miR-432 resulted in a significant decrease in FN1 expression in HeLa and SiHa cells at the mRNA $(\mathrm{P}<0.05$; Fig. 3E) and protein ( $\mathrm{P}<0.05$; Fig. 3F) levels. Collectively, these results indicate that FN1 is a direct target gene of miR-432 in cervical cancer cells.

Inhibition of FN1 stimulates the tumour suppressor activity of miR-432 in cervical cancer cells. To clarify the biological roles of FN1 in cervical cancer, FN1 siRNA was introduced into HeLa and SiHa cells to decrease FN1 expression. The protein level of FN1 was efficiently knocked down in HeLa and SiHa cells following FN1 siRNA transfection $(\mathrm{P}<0.05$; Fig. 4A). CCK-8 and Transwell Matrigel invasion assays were performed to identify the effects of FN1 inhibition on the proliferation and invasion of cervical cancer cells, respectively. FN1 silencing significantly decreased proliferation at 48 and $72 \mathrm{~h}(\mathrm{P}<0.05$; Fig. 4B) and invasion $(\mathrm{P}<0.05$; Fig. 4C) of HeLa and SiHa cells compared with the NC siRNA cells. These results indicated that the functional roles of FN1 inhibition were similar to those induced by miR-432 upregulation in cervical cancer cells, further identifying FN1 as a downstream target of miR-432.

FN1 restoration rescues the suppressive effects of miR-432 on the malignant phenotypes of cervical cancer cells. miR-432 overexpression suppressed the proliferation and invasion of cervical cancer cells, and FN1 was identified as a direct target gene of miR-432, therefore it was 

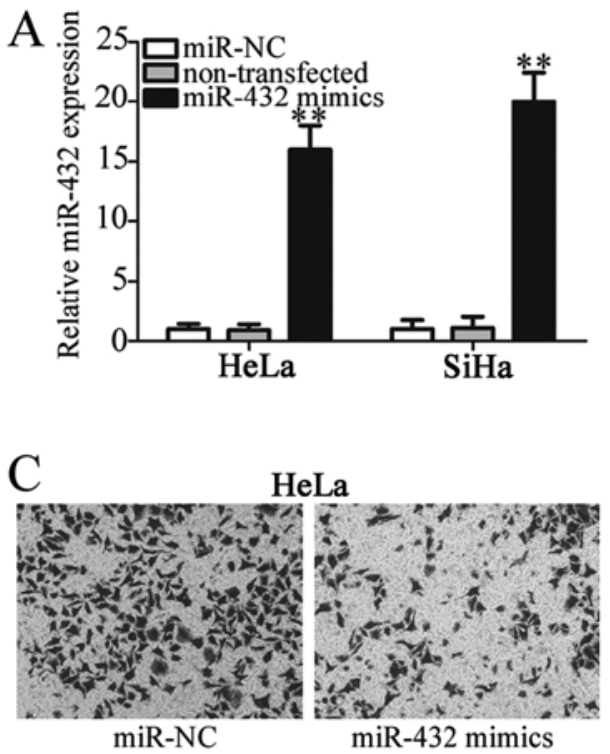
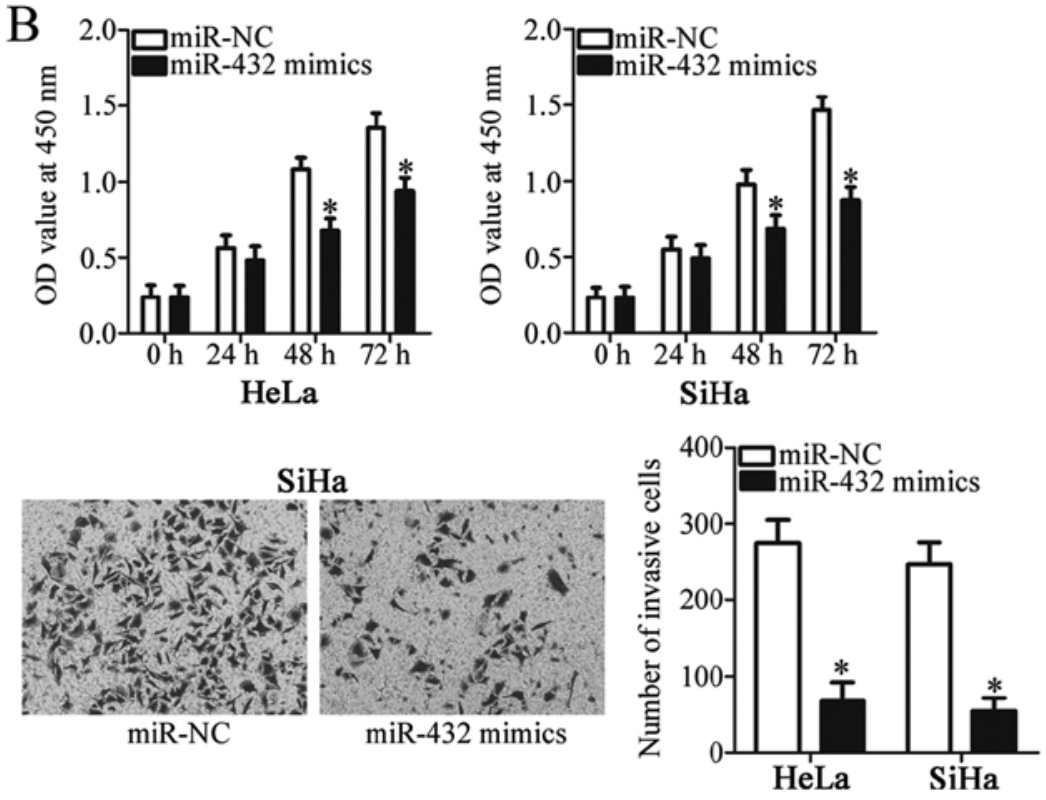

Figure 2. miR-432 upregulation restricts the proliferation and invasion of HeLa and SiHa cells. (A) HeLa and SiHa cells were transfected with miR-432 mimic or miR-NC. At $48 \mathrm{~h}$ after transfection, RT-qPCR was used to detect miR-432 expression. ${ }^{*} \mathrm{P}<0.05$ vs. miR-NC and non-transfected cells. (B) A Cell Counting Kit-8 assay was performed to investigate cellular proliferation of HeLa and SiHa cells following miR-432 mimic or miR-NC transfection. (C) The invasive ability of miR-432 mimic or miR-NC-transfected HeLa and SiHa cells was investigated using a Transwell Matrigel invasion assay. * $<0.05$ vs. miR-NC. miR-432, microRNA-432; RT-qPCR, reverse transcription-quantitative polymerase chain reaction; NC, negative control; OD, optical density.

A

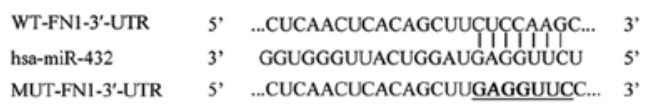

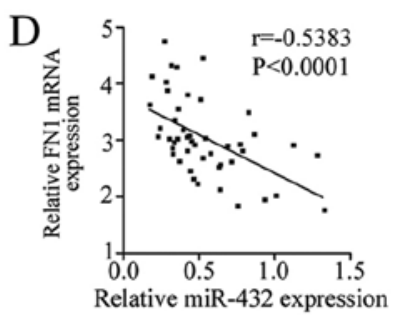

B
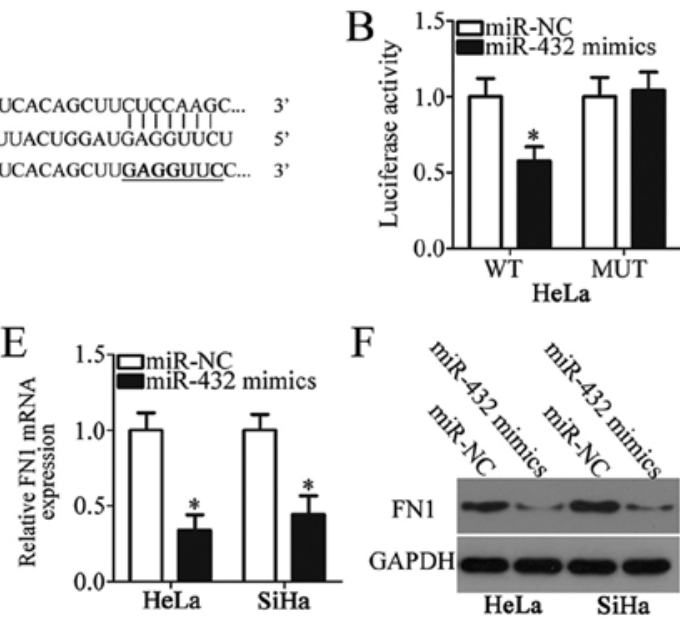

$\mathrm{F}$

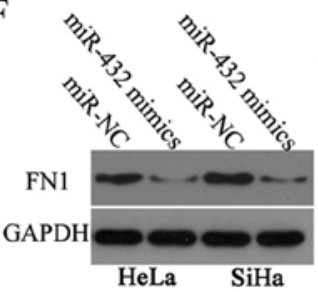

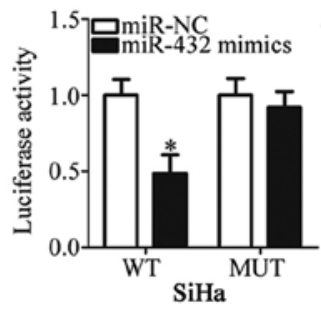
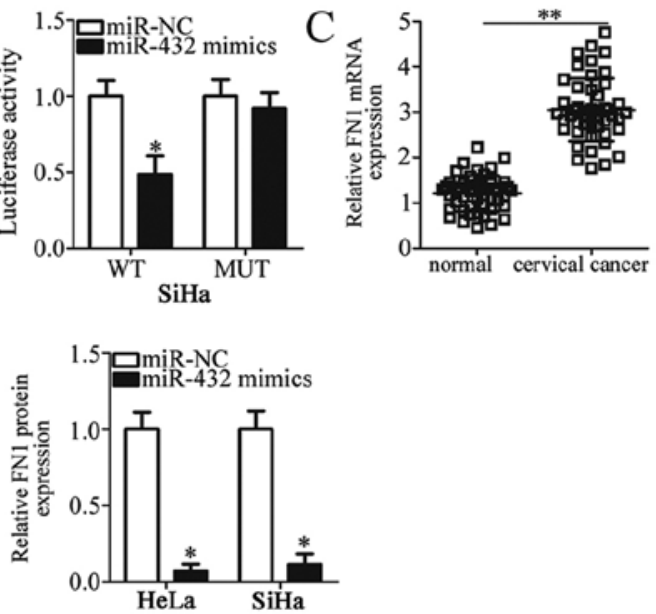

Figure 3.FN1 is a direct target gene of miR-432 in cervical cancer cells. (A) The WT binding sequences of miR-432 in the 3'-UTR of FN1 as predicted by a bioinformatics analysis. The MUT binding site was also generated in the seed region of the 3'-UTR of FN1.(B) pMIR-WT-FN1-3'-UTR or pMIR-MUT-FN1-3'-UTR was co-transfected with miR-432 mimic or miR-NC into HeLa and SiHa cells. Cells were collected and used for the detection of luciferase activity $48 \mathrm{~h}$ after transfection. (C) RT-qPCR was performed to determine the expression level of FN1 mRNA in cervical cancer tissues and matched adjacent normal tissues. (D) Spearman's correlation analysis was performed to investigate the correlation between miR-432 and FN1 mRNA levels in cervical cancer tissues. $r=-0.5383$, $\mathrm{P}<0.0001$ (E) FN1 mRNA expression in HeLa and SiHa cells following transfection with miR-432 mimic or miR-NC was measured by RT-qPCR and western blot analysis. (F) Protein expression in HeLa and SiHa cells following transfection with miR-432 mimic or miR-NC was measured using western blot analysis. "P<0.05 vs. miR-NC; ${ }^{* *} \mathrm{P}<0.05$ vs. adjacent normal tissues. FN1, fibronectin 1; miR-432, microRNA-432; WT, wild-type; UTR, untranslated region; MUT, mutated; NC, negative control; RT-qPCR, reverse transcription-quantitative polymerase chain reaction.

hypothesized that FN1 downregulation was essential for the miR-432-mediated malignant phenotypes of cervical cancer cells. To confirm this hypothesis, miR-432 mimics along with pcDNA3.1-FN1 (pc-FN1) or blank pcDNA3.1 plasmid were co-transfected into $\mathrm{HeLa}$ and $\mathrm{SiHa}$ cells. Following transfection, western blot analysis revealed that the decreased level of FN1 protein in HeLa and SiHa cells caused by miR- 432 overexpression was almost recovered by co-transfection with pc-FN1 ( $\mathrm{P}<0.05$; Fig. 5A). CCK-8 and Transwell Matrigel invasion assays were further performed. The proliferation and invasion of $\mathrm{HeLa}$ and $\mathrm{SiHa}$ cells were significantly suppressed upon miR-432 overexpression; however, the inhibition of proliferation $(\mathrm{P}<0.05$; Fig. $5 \mathrm{~B})$ and invasion $(\mathrm{P}<0.05$; Fig. $5 \mathrm{C})$ associated with miR-432 overexpression were reversed by FN1 restoration. This suggests that the tumour suppressive roles of miR-432 in 
A

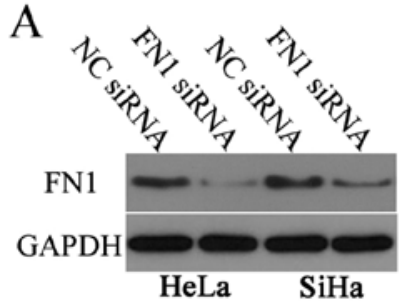

$\mathrm{C}$

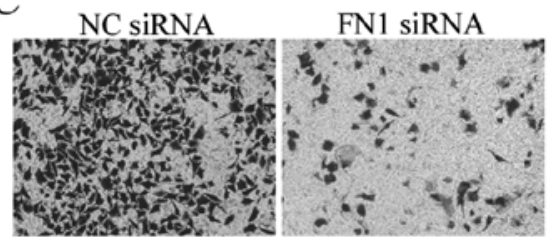

$\mathrm{HeLa}$
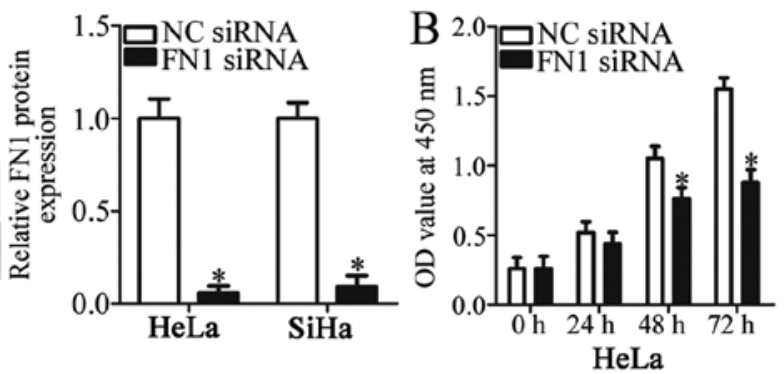

HeLa

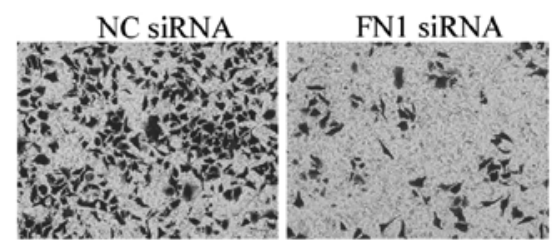

$\mathrm{SiHa}$
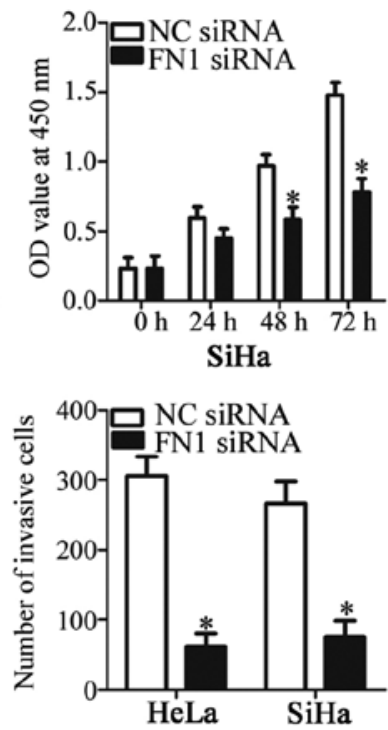

Figure 4. FN1 silencing suppresses the cellular proliferative and invasive capacities of HeLa and CaSki cells. (A) FN1 protein level in HeLa and SiHa cells treated with FN1 siRNA or NC siRNA was detected using the reverse transcription-quantitative polymerase chain reaction. (B) Cell Counting Kit-8 was used to assess the proliferation of HeLa and SiHa cells upon FN1 inhibition. (C) Transwell Matrigel invasion assay was used to assess the invasion of HeLa and SiHa cells upon FN1 inhibition. "P<0.05 vs. NC siRNA. FN1, fibronectin 1; siRNA, small interfering RNA; NC, negative control; OD, optical density.
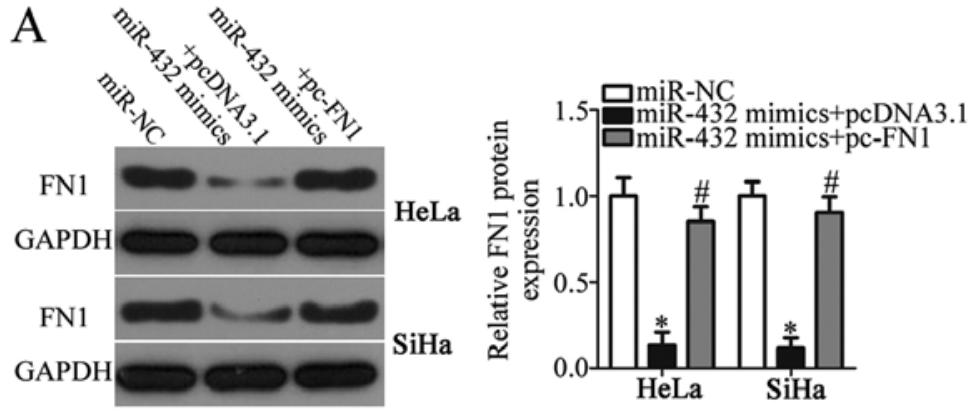

$\mathrm{C}$

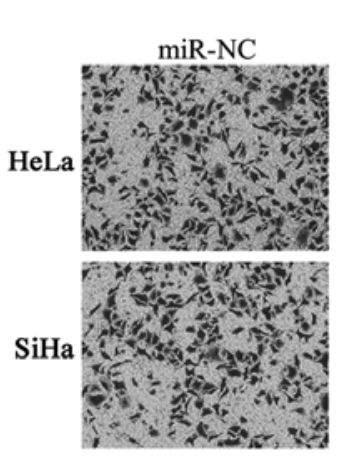

miR-432 mimics + pcDNA3.1

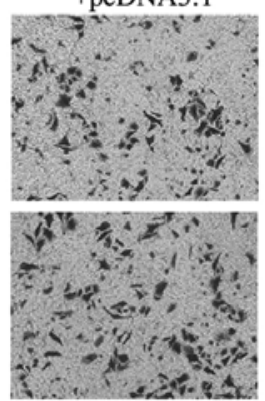

B
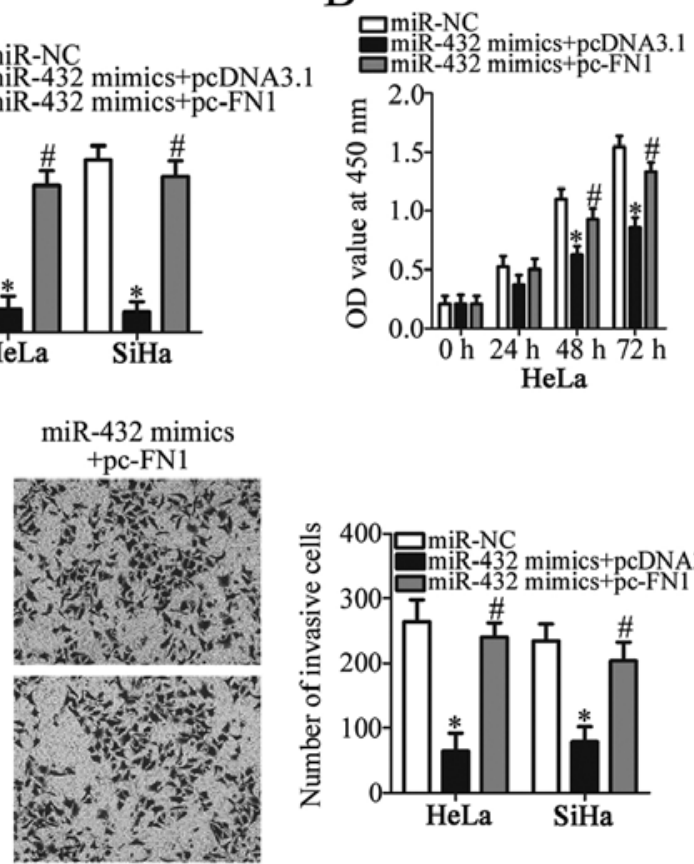
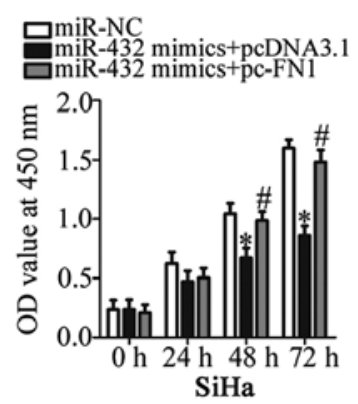

Figure 5. Recovered FN1 expression attenuates the inhibitory effects of miR-432 overexpression in the proliferation and invasion of HeLa and SiHa cells. miR-432 mimic along with FN1 overexpression plasmid pcDNA3.1-FN1 or pcDNA3.1 was introduced into HeLa and SiHa cells. (A) Western blot analysis was performed to determine the expression of FN1 protein. (B and C) The proliferation of the aforementioned cells was determined using a Cell Counting Kit-8. (C) The invasion of the aforementioned cells was determined by performing a Transwell Matrigel invasion assay. " $\mathrm{P}<0.05$ vs. miR-NC; ${ }^{\mathrm{P}}<0.05$ vs. miR-432 mimics+pcDNA3.1. FN1, fibronectin 1; miR-432, microRNA-432; OD, optical density.

cervical cancer cells may be, at least in part, attributable to the downregulation of FN1.

\section{Discussion}

Numerous studies have demonstrated the dysregulation of miRNAs in cervical cancer (28-30). miRNAs serve as oncogenes or tumour suppressors and are involved in regulating a number of tumour-associated biological behaviours, including the cell cycle, cell proliferation, apoptosis and metastasis (2). Therefore, investigating the roles of cervical cancer-associated miRNAs and the underlying molecular mechanisms is essential for the development of novel diagnostic biomarkers and effective therapeutic targets. In the 
present study, the expression levels and clinical value of miR-432 in cervical cancer were investigated. Furthermore, the detailed roles of miR-432 in the development of cervical cancer were determined. Notably, the underlying molecular mechanisms by which miR-432 may affect the development of cervical cancer were investigated.

miR-432 is deregulated in several types of human cancer. For instance, miR-432 was downregulated in lung adenocarcinoma tissues and cell lines. Downregulation of miR-432 was significantly associated with the clinical stage of patients with lung adenocarcinoma. Patients with lung adenocarcinoma that have low miR-432 levels presented with poorer prognoses than those patients with high miR-432 levels (18). miR-432 was also expressed at low levels in neuroblastoma (19), hepatocellular carcinoma (20) and prostate cancer (21). However, the expression status of miR-432 in cervical cancer remains unclear. In the present study, RT-qPCR analysis was used to detect miR-432 expression in cervical cancer tissues and cell lines. The results of the present study revealed that miR-432 was expressed at low levels in cervical cancer tissues and cell lines. In addition, decreased miR-432 expression was associated with FIGO stage, myometrium invasion and lymph node metastasis in patients with cervical cancer. These results suggest that miR-432 may be a potential biomarker for the diagnosis of patients with these specific types of cancer.

miR-432 deregulation contributes to the carcinogenesis and progression of several types of human malignancy. For example, miR-432 re-expression repressed cell proliferation and induced cell cycle arrest in lung adenocarcinoma. In addition, miR-432 overexpression increased the chemosensitivity of lung adenocarcinoma cells to cisplatin (18). In neuroblastoma, enforced miR-432 expression decreased cell proliferation, promoted G0-G1 cell cycle arrest and induced neurite projections (19). In hepatocellular carcinoma, restoration of miR-432 expression inhibited cell proliferation and colony formation, increased G0-G1 cell cycle arrest in vitro and decreased tumour growth in vivo through deactivation of the Wnt/ $\beta$-catenin signalling pathway (20). In prostate cancer, ectopic miR-432 expression inhibited the activation of the Wnt $/ \beta$-catenin signalling pathway and participated in the regulation of cell proliferation and apoptosis. Nevertheless, to the best of our knowledge, the functional roles of miR-432 in the progression and development of cervical cancer have not been investigated. In the present study, functional experiments demonstrated that resumption of miR-432 expression restrained the proliferation and invasion of cervical cancer cells. These results suggest that miR-432 may represent a useful therapeutic target for managing patients with these types of malignant tumour.

Several genes have been identified as direct targets of miR-432, including E2F transcription factor 3 and anexelekto in lung adenocarcinoma (18), nestin and REST corepressor 1 in neuroblastoma (19) and tripartite motif-containing protein 29 and Pygopus homolog 2 in prostate cancer (21). In the present study, the underlying molecular mechanism by which miR-432 regulates the development of cervical cancer was also investigated. FN1, a member of the FN family (24), was identified as a direct target of miR-432 in cervical cancer. The expression of FN1 was previously reported to be upregulated in numerous types of human malignant tumours, including breast cancer (31), gastric cancer (32), ovarian cancer (33) and thyroid cancer (34). A number of studies have demonstrated the key role that FN1 serves in tumourigenesis and tumour progression; FN1 serves oncogenic roles and regulates a variety of cellular processes (24-27). In the present study, it was revealed that FN1 was markedly expressed in cervical cancer, and that inhibition of FN1 could inhibit the proliferation and invasion of cervical cancer cells. These data suggest that targeting FN1 may be a promising approach for treating patients with cervical cancer.

In conclusion, miR-432 was downregulated in cervical cancer, which was correlated with FIGO stage, myometrium invasion and lymph node metastasis. Enforced miR-432 expression suppressed the proliferation and invasion of cervical cancer cells by directly targeting FN1. The results of the present study provide a theoretical basis for the application of the miR-432/FN1 pathway in the treatment of patients with cervical cancer. However, there were two limitations to the present study: First, the influence of miR-432 on the cell cycle of cervical cancer was not investigated. Secondly, the sample size of the present study was small. Future studies will aim to resolve these two limitations.

\section{Acknowledgements}

Not applicable.

\section{Funding}

No funding was received.

\section{Availability of data and materials}

The datasets used and/or analysed during the present study are available from the corresponding author upon reasonable request.

\section{Authors' contributions}

WW and SW designed this study. SW and BG performed RT-qPCR, CCK-8 assay and luciferase reporter assay. Transwell Matrigel invasion assay was carried out by HY. XL and XW conducted western blot analysis. All authors have made a significant contribution to the findings and methods, and have read and approved the final manuscript.

\section{Ethics approval and consent to participate}

The present study was approved by the Ethics Committee of The Third People's Hospital of Linyi (Linyi, China). All patients provided written informed consent.

\section{Patient consent for publication}

Not applicable.

\section{Competing interests}

The authors declare that they have no competing interests. 


\section{References}

1. Torre LA, Bray F, Siegel RL, Ferlay J, Lortet-Tieulent J and Jemal A: Global cancer statistics, 2012. CA Cancer J Clin 65: 87-108, 2015

2. Banno K, Iida M, Yanokura M, Kisu I, Iwata T, Tominaga E, Tanaka K and Aoki D: MicroRNA in cervical cancer: OncomiRs and tumor suppressor miRs in diagnosis and treatment. ScientificWorldJournal 2014: 178075, 2014.

3. Siegel R, Naishadham D and Jemal A: Cancer statistics, 2012. CA Cancer J Clin 62: 10-29, 2012

4. Handler AS, Henderson VA, Rosenfeld A, Rankin K, Jones B and Issel LM: Illinois breast and cervical cancer program: Implementing effective public-private partnerships to assure population health. J Public Health Manag Pract 21: 459-466, 2015

5. Kogo R, How C, Chaudary N, Bruce J, Shi W, Hill RP, Zahedi P, Yip KW and Liu FF: The microRNA-218 Survivin axis regulates migration, invasion, and lymph node metastasis in cervical cancer. Oncotarget 6: 1090-1100, 2015.

6. Griffiths-Jones S, Grocock RJ, van Dongen S, Bateman A and Enright AJ: miRBase: microRNA sequences, targets and gene nomenclature. Nucleic Acids Res 34: D140-D144, 2006.

7. Shu L, Zhang Z and Cai Y: MicroRNA-204 inhibits cell migration and invasion in human cervical cancer by regulating transcription factor 12. Oncol Lett 15: 161-166, 2018.

8. Srivastava SK, Ahmad A, Zubair H, Miree O, Singh S, Rocconi RP, Scalici J and Singh AP: MicroRNAs in gynecological cancers: Small molecules with big implications. Cancer Lett 407: 123-138, 2017.

9. Zhao Y, Liu X and Lu YX: MicroRNA-143 regulates the proliferation and apoptosis of cervical cancer cells by targeting HIF-1alpha. Eur Rev Med Pharmacol Sci 21: 5580-5586, 2017.

10. Zhang H, Lu Y, Wang S, Sheng X and Zhang S: MicroRNA-152 acts as a tumor suppressor microRNA by inhibiting Kruppel-like factor 5 in human cervical cancer. Oncol Res 27: 335-340, 2019.

11. Gong M, Chen C, Zhao H, Sun M and Song M: miR-506 suppresses cervical cancer cell proliferation both in vitro and in vivo. Neoplasma 65: 331-338, 2018

12. Zhang Z, Wang J, Wang X, Song W, Shi Y and Zhang L: MicroRNA-21 promotes proliferation, migration, and invasion of cervical cancer through targeting TIMP3. Arch Gynecol Obstet 297: 433-442, 2018

13. Yu LM, Wang WW, Qi R, Leng TG and Zhang XL: MicroRNA-224 inhibition prevents progression of cervical carcinoma by targeting PTX3. J Cell Biochem 119: 10278-10290, 2018.

14. Chen Z, Zhang M, Qiao Y, Yang J and Yin Q: MicroRNA-1297 contributes to the progression of human cervical carcinoma through PTEN. Artif Cells Nanomed Biotechnol 46 (Suppl 2): S1120-S1126, 2018.

15. Kanekura K, Nishi H, Isaka K and Kuroda M: MicroRNA and gynecologic cancers. J Obstet Gynaecol Res 42: 612-617, 2016.

16. Gonzalez-Quintana V, Palma-Berré L, Campos-Parra AD, López-Urrutia E, Peralta-Zaragoza O, Vazquez-Romo R and Pérez-Plasencia C: MicroRNAs are involved in cervical cancer development, progression, clinical outcome and improvement treatment response (Review). Oncol Rep 35: 3-12, 2016.

17. Wang F, Li B and Xie X: The roles and clinical significance of microRNAs in cervical cancer. Histol Histopathol 31: 131-139, 2016.

18. Chen L, Kong G, Zhang C, Dong H, Yang C, Song G, Guo C, Wang $\mathrm{L}$ and $\mathrm{Yu} \mathrm{H}$ : MicroRNA-432 functions as a tumor suppressor gene through targeting E2F3 and AXL in lung adenocarcinoma. Oncotarget 7: 20041-20053, 2016.

19. Das E and Bhattacharyya NP: MicroRNA-432 contributes to dopamine cocktail and retinoic acid induced differentiation of human neuroblastoma cells by targeting NESTIN and RCOR1 genes. FEBS Lett 588: 1706-1714, 2014.
20. Jiang N, Chen WJ, Zhang JW, Xu C, Zeng XC, Zhang T, Li Y and Wang GY: Downregulation of miR-432 activates Wnt/beta-catenin signaling and promotes human hepatocellular carcinoma proliferation. Oncotarget 6: 7866-7879, 2015.

21. Li JB, Liu F, Zhang BP, Bai WK, Cheng W, Zhang YH and Yu LJ: LncRNA625 modulates prostate cancer cells proliferation and apoptosis through regulating the Wnt/beta-catenin pathway by targeting miR-432. Eur Rev Med Pharmacol Sci 21: 2586-2595, 2017.

22. Guo L, Liu X, Wang L, Sun H, Huang K, Li X, Tang F, Li S, Yuan X and Wang C: Outcome of international Federation of gynecology and obstetrics stage IIb cervical cancer from 2003 to 2012: An evaluation of treatments and prognosis: A retrospective study. Int J Gynecol Cancer 25: 910-918, 2015.

23. Livak KJ and Schmittgen TD: Analysis of relative gene expression data using real-time quantitative PCR and the 2(-Delta Delta C(T)) method. Methods 25: 402-408, 2001

24. Cai X, Liu C, Zhang TN, Zhu YW, Dong X and Xue P. Down-regulation of FN1 inhibits colorectal carcinogenesis by suppressing proliferation, migration, and invasion. J Cell Biochem 119: 4717-4728, 2018.

25. Ye Y, Zhuang J, Wang G, He S, Ni J and Xia W: MicroRNA-139 targets fibronectin 1 to inhibit papillary thyroid carcinoma progression. Oncol Lett 14: 7799-7806, 2017.

26. Wang J, Deng L, Huang J, Cai R, Zhu X, Liu F, Wang Q, Zhang J and Zheng Y: High expression of Fibronectin 1 suppresses apoptosis through the NF- $\mathrm{B}$ pathway and is associated with migration in nasopharyngeal carcinoma. Am J Transl Res 9: 4502-4511, 2017

27. Xu X, Liu Z, Zhou L, Xie H, Cheng J, Ling Q, Wang J, Guo H, Wei X and Zheng S: Characterization of genome-wide TFCP2 targets in hepatocellular carcinoma: Implication of targets FN1 and TJP1 in metastasis. J Exp Clin Cancer Res 34: 6, 2015.

28. Diaz-Gonzalez Sdel M, Deas J, Benitez-Boijseauneau O, Gómez-Cerón C, Bermúdez-Morales VH, Rodríguez-Dorantes M, Pérez-Plasencia $\mathrm{C}$ and Peralta-Zaragoza O: Utility of microRNAs and siRNAs in cervical carcinogenesis. Biomed Res Int 2015: 374924, 2015 .

29. Yang F, Guo L, Cao Y, Li S, Li J and Liu M: MicroRNA-7-5p promotes cisplatin resistance of cervical cancer cells and modulation of cellular energy homeostasis by regulating the expression of the PARP-1 and BCL2 genes. Med Sci Monit 24: 6506-6516, 2018.

30. Shang A, Zhou C, Bian G, Chen W, Lu W, Wang W and Li D: miR-381-3p restrains cervical cancer progression by downregulating FGF7. J Cell Biochem 120: 778-789, 2019.

31. Ruiz-Garcia E, Scott V, Machavoine C, Bidart JM, Lacroix L, Delaloge S and Andre F: Gene expression profiling identifies Fibronectin 1 and CXCL9 as candidate biomarkers for breast cancer screening. Br J Cancer 102: 462-468, 2010.

32. Zhang H, Sun Z, Li Y, Fan D and Jiang H: MicroRNA-200c binding to FN1 suppresses the proliferation, migration and invasion of gastric cancer cells. Biomed Pharmacother 88: 285-292, 2017.

33. Helleman J, Jansen MP, Span PN, van Staveren IL, Massuger LF, Meijer-van Gelder ME, Sweep FC, Ewing PC, van der Burg ME, Stoter $\mathrm{G}$, et al: Molecular profiling of platinum resistant ovarian cancer. Int J Cancer 118: 1963-1971, 2006.

34. Sponziello M, Rosignolo F, Celano M, Maggisano V, Pecce V, De Rose RF, Lombardo GE, Durante C, Filetti S, Damante G, et al: Fibronectin-1 expression is increased in aggressive thyroid cancer and favors the migration and invasion of cancer cells. Mol Cell Endocrinol 431: 123-132, 2016. 